\title{
L-Leucine increases the daily body temperature and affords thermotolerance in broiler chicks
}

\author{
Guofeng Han', Hui Yang' ${ }^{1}$ Yunhao Wang', Shogo Haraguchi², Takuro Miyazaki², Takashi Bungo ${ }^{3}$, \\ Kosuke Tashiro ${ }^{4}$, Mitsuhiro Furuse ${ }^{1}$, and Vishwajit S. Chowdhury ${ }^{1,5, *}$
}

\author{
* Corresponding Author: Vishwajit S. Chowdhury \\ Tel: +81-0-92-802-6015, Fax: +81-0-92-802-6009, \\ E-mail:vc-sur@artsci.kyushu-u.ac.jp \\ ${ }^{1}$ Graduate School of Bioresource and \\ Bioenvironmental Sciences, Kyushu University, \\ Fukuoka 819-0395, Japan \\ 2 Department of Biochemistry, Showa University School \\ of Medicine, Tokyo 152-8555, Japan \\ ${ }^{3}$ Department of Bioresource Science, Hiroshima \\ University, Higashi-Hiroshima 739-8528, Japan \\ ${ }^{4}$ Department of Molecular Biosciences, Faculty of \\ Agriculture, Kyushu University, Fukuoka 819-0395, \\ Japan \\ ${ }^{5}$ Faculty of Arts and Science, Kyushu University, \\ Fukuoka 819-0395, Japan \\ ORCID \\ Guofeng Han \\ https://orcid.org/0000-0002-3685-4062 \\ Hui Yang \\ https://orcid.org/0000-0001-9512-2665 \\ Yunhao Wang \\ https://orcid.org/0000-0002-1384-0223 \\ Shogo Haraguchi \\ https://orcid.org/0000-0002-8731-3311 \\ Takuro Miyazaki \\ https://orcid.org/0000-0003-3354-0292 \\ Takashi Bungo \\ https://orcid.org//0000-0002-3575-0974 \\ Kosuke Tashiro \\ https://orcid.org/0000-0003-4980-2058 \\ Mitsuhiro Furuse \\ https://orcid.org/0000-0002-7474-1165 \\ Vishwajit S. Chowdhury \\ https://orcid.org/0000-0001-6709-2788
}

Submitted Sept 7, 2018; Revised Oct 8, 2018; Accepted Oct 23, 2018
Objective: Heat stress poses an increasing threat for poultry production. Some amino acids have been found to play critical roles in affording thermotolerance. Recently, it was found that in ovo administration of L-leucine (L-Leu) altered amino acid metabolism and afforded thermotolerance in heat-exposed broiler chicks.

Methods: In this study, two doses ( 35 and $70 \mu \mathrm{mol} / \mathrm{egg}$ ) of L-Leu were administered in ovo on embryonic day 7 to determine their effect on rectal temperature (RT), body weight (BW) and thyroid hormones at hatching. Changes in RT, BW, and thermotolerance in post-hatched chicks were also analyzed.

Results: It was found that in ovo administration of L-Leu dose-dependently reduced RT and plasma thyroxine $\left(\mathrm{T}_{4}\right)$ level just after hatching. In post-hatched neonatal broiler chicks, however, the higher dose of L-Leu administered in ovo significantly increased RT without affecting BW gain. In chicks that had been exposed to heat stress, the RT was significantly lowered by in ovo administration of L-Leu (high dose) in comparison with the control chicks under the same high ambient temperature ( $\left.\mathrm{HT}: 35^{\circ} \mathrm{C} \pm 1^{\circ} \mathrm{C}, 120 \mathrm{~min}\right)$.

Conclusion: In ovo administration of L-Leu in a high dose contributed to an increased daily body temperature and afforded thermotolerance under HT in neonatal broiler chicks.

Keywords: L-leucine; Chicks; Heat Stress; Rectal Temperature; Thermotolerance

\section{INTRODUCTION}

Heat stress presents an increasing challenge for poultry production across the world since chickens are very sensitive to high ambient temperature (HT) [1]. The HT causes their body temperature to increase and induces oxidative stress [2,3]. Promoting the development of thermotolerance in chickens would therefore be one a timely approach to dealing with this challenge. Some free amino acids are recognized as biomarkers of heat stress, since they are significantly affected by heat stress $[3,4]$. These free amino acids play critical roles in thermoregulation, as well as in reducing body temperature in chicks under heat stress [5-9].

Leucine (Leu) has been demonstrated to play critical roles in physiological regulation, including in protein synthesis, in reducing muscle damage, and in glucose or lipid metabolic activity $[10,11]$. In our previous study, it was found that thermal manipulation (TM), which is a technique enabling the acquisition of thermotolerance in chickens [12], significantly decreased brain and hepatic Leu concentration in developing embryos [7]. In ovo administration of L-Leu ( $35 \mu \mathrm{mol} / \mathrm{egg}$ ) on embryonic day (ED) 7 was then found to cause hypothermia at hatching, and also to afford thermotolerance in young broiler male chicks under HT [7,8]. However, the appropriate dose at which to administer L-Leu in ovo to develop thermoregulatory functions in broiler chicks is still unknown. The thyroid hormones thyroxine $\left(\mathrm{T}_{4}\right)$ and 
triiodothyronine $\left(\mathrm{T}_{3}\right)$ are associated with metabolic rate, which affects the regulation of body temperature $[13,14]$. It was concluded that TM during embryogenesis caused the thyroid and adrenal hormone levels to decline, resulting in hypothermia at hatching [15]. The first aim of this study was to compare the effects on body temperature and body weight (BW) of two doses (35 or $70 \mu \mathrm{mol} / \mathrm{egg}$ ) of L-Leu administered in ovo, as well as their effects on thyroid hormone levels at hatching in broiler chicks.

Plasma triacylglycerol, non-esterified fatty acid (NEFA) and ketone body levels were significantly increased, and in addition, food intake significantly decreased, in male chicks administered with L-Leu in ovo compared with control chicks at 180 min of a heat challenge $\left(35^{\circ} \mathrm{C} \pm 1^{\circ} \mathrm{C}\right)[8]$. The stimulated lipid metabolic activity was suggested as a possible endogenous factor contributing to the development of thermotolerance under heat stress in broiler chicks [8]. Therefore, it is important to examine the plasma metabolites. In ovo administration of a high dose of L-Leu could be expected to produce more potential effects of L-Leu in terms of affording thermotolerance in broilers and it could also help to clarify the mechanisms whereby L-Leu develops heat-resistant broilers. The second aim of this study was to investigate the effect of in ovo administration of a high dose $(70 \mu \mathrm{mol} / \mathrm{egg})$ of L-Leu on daily body temperature and BW, as well as on food intake, plasma metabolites and thermotolerance in chicks under heat stress.

\section{MATERIALS AND METHODS}

\section{Experimental design}

In Experiment 1, forty-five fertilized broiler eggs were purchased from a local hatchery (Mori Hatchery, Fukuoka, Japan) and placed in an incubator (SHOWA P008-type incubator, SHOWA Furanki Company, Saitama, Japan). The eggs were divided into three groups ( $\mathrm{n}=15$ /group) based on the initial egg weight, with the resulting groups being as uniform possible. The groups were as follows: control (sterile water injection in the amount of $500 \mu \mathrm{L} / \mathrm{egg})$; L-Leu low dose $(35 \mu \mathrm{mol} / 500$ $\mu \mathrm{L} /$ egg); and L-Leu high dose $(70 \mu \mathrm{mol} / 500 \mu \mathrm{L} / \mathrm{egg})$ (average egg weight: control $=65.2 \mathrm{~g}$; L-Leu (low) $=65.2 \mathrm{~g}$ : L-Leu (high) $=65.3 \mathrm{~g}$ ). The eggs were incubated at a temperature of $37.6^{\circ} \mathrm{C}$, at $58 \%$ to $68 \%$ relative humidity, and with auto-turning per h. The process of in ovo administration was the same as reported previously $[7,8]$. In brief, an injection was performed on ED 7. Unfertilized eggs were detected by candling and discarded prior to the injection. After sterilization, a small hole was made at the large end of the egg and sterile water or L-Leu solution was injected to a depth of $25 \mathrm{~mm}$ with a 1-mL disposable syringe that had a 25 -gauge needle. The small holes were immediately sealed with Scotch tape after injection. At ED 18, the eggs were shifted to the hatching trays after detecting the undeveloped and dead embryos by candling and removing them.
Rectal temperature (RT) and BW were recorded at $2 \mathrm{~h}$ after hatching. The RT was recorded by a digital thermometer with an accuracy of $\pm 0.1^{\circ} \mathrm{C}$ (Thermalert TH-5, Physitemp Instruments Inc., Clifton, NJ, USA), by inserting the thermistor probe into the colon (rectum) through the cloaca to a depth of $2 \mathrm{~cm}$, as we reported previously $[7,16]$. The chicks were properly anesthetized with isoflurane (Mylan Inc., Tokyo, Japan) before a blood sample was collected from the jugular vein. The blood was collected in heparinized tubes and centrifuged at $10,000 \times g$ at $4^{\circ} \mathrm{C}$ for $4 \mathrm{~min}$ in order to separate out the plasma.

In Experiment 2, fifty-four eggs were divided into two groups based on their initial weight. The experimental groups were: control $(n=27$; average egg weight $=64.1 \mathrm{~g})$ and L-Leu high dose $(n=27$; average egg weight $=63.9 \mathrm{~g})$. The incubation and in ovo administration processes were the same as described above in Experiment 1. After hatching, the chicks were housed in groups in metal cages (floor space: $36 \mathrm{~cm} \times 50 \mathrm{~cm}$; height: $30 \mathrm{~cm}$ ) under a control thermoneutral temperature $(\mathrm{CT})$ with the following temperature schedule: $30^{\circ} \mathrm{C}$ for 1 to 4 days, and $28^{\circ} \mathrm{C}$ for 5 to 10 days. The chicks were provided with free access to food (Adjust diets; metabolizable energy $>12.6 \mathrm{MJ} / \mathrm{kg}$ and crude protein $>23 \%$; Toyohashi Feed and Mills Co. Ltd., Aichi, Japan) and water as well as continuous light. In previous studies [7,8], in ovo administration of L-Leu was found to afford thermotolerance in young male broiler chicks. At 2 days old, therefore, the males were separated, by means of sexing through feather identification, for further experimental procedures to be carried out on them. The 19 males (control $=10$; L-Leu high dose $=9$ ) were subjected to a post-hatch experiment. At 8 days old, the males were individually isolated in small metal cages (floor space: $20 \mathrm{~cm} \times 28 \mathrm{~cm}$; height: $30 \mathrm{~cm}$ ). Since in our previous study the body temperature in birds administered with L-Leu in ovo was found to have significantly decreased $120 \mathrm{~min}$ after starting the heat stress during $180 \mathrm{~min}$ of heat exposure [8], in the current study we decided to set a time point of $120 \mathrm{~min}$, when we would examine the effects of heat stress. At 10 days old, the male chicks from each group were randomly exposed to $\mathrm{HT}\left(35^{\circ} \mathrm{C} \pm 1^{\circ} \mathrm{C}\right)$ for $120 \mathrm{~min}$. The heat-exposed chicks were put into two chambers (Sanyo Electric Co. Ltd., Osaka, Japan; Catalog number: Sanyo MIR-254). All birds were provided with free access to food and water under HT. Food intake was measured on the basis of the amount of food that had disappeared over $120 \mathrm{~min}$ in the feeders. Adequate precautions were taken against food spillage - namely, the feeders were only half-filled so that chicks could not spill any food. RT was recorded at 0 min and 120 min of heat exposure. Plasma was collected following proper anesthetization with isoflurane (Mylan Inc., Japan), as in Experiment 1 . All the collected samples were stored at $-80^{\circ} \mathrm{C}$ until further analysis took place.

This study was performed in accordance with the guidelines for animal experiments in the Faculty of Agriculture of Kyushu 
University and complied with Law No. 105 and Notification No. 6 of the Japanese government.

\section{Analysis of plasma thyroid hormones}

Plasma samples were subjected to LC-MS/MS analysis to determine $\mathrm{T}_{3}$ and $\mathrm{T}_{4}$ concentrations. Briefly, plasma samples were spiked with ${ }^{13} \mathrm{C}_{6}$-T3 (T-087, Sigma, St. Louis, MO, USA) as an internal standard and were added to a mixture of $5 \% \mathrm{NH}_{4} \mathrm{OH}$ and acetonitrile $(50 / 50,[\mathrm{v} / \mathrm{v}])$, and mixed thoroughly in a vortex. Samples were loaded onto SPE columns (EVOLUTE EXPRESS AX, Biotage, Charlotte, NC, USA) and extracted by using $5 \%$ formic acid in methanol. After evaporation, the samples were reconstituted in $2 \mathrm{mM}$ ammonium acetate/ $0.1 \%$ formic acid/50\% methanol for LC-MS/MS analysis. The samples were analyzed on a QTRAP 5500 LC-MS/MS system (AB SCIEX, Tokyo, Japan) connected to a Shimadzu LC 20A HPLC system. The multiple reaction monitoring transitions in positive ionization mode were as follows: for $\mathrm{T}_{3}, 651.8>605.8$; for $\mathrm{T}_{4}, 777.8>731.8$; for ${ }^{13} \mathrm{C}_{6}-\mathrm{T} 3,657.9>611.9$.

\section{Analysis of plasma metabolites}

Plasma NEFA, ammonia $\left(\mathrm{NH}_{3}\right)$, lactic acid (LA), lactate dehydrogenase (LDH), immunoglobulin A (IgA) and ketone bodies were examined using a reagent kit provided by the manufacturer to run in a Beckman Coulter AU480 automatic biochemistry analysis system (Beckman Coulter, Brea, CA, USA).

\section{Statistical analyses}

The data concerning RT and relative BW, as well as thyroid hormones at hatching, were statistically analyzed by one-way analysis of variance (ANOVA) following a post-hoc analysis using Holm-Sidak's multiple comparisons test that was carried out when a significant difference was detected. The data concerning daily RT and BW, as well as RT changes under heat stress, were statistically analyzed by a two-way repeated-measures ANOVA following a post-hoc analysis using Holm-Sidak's multiple comparisons test that was carried out when a significant interaction was detected, where the main effects were in ovo administration of L-Leu and age or HT. The data concerning food intake under heat stress were statistically analyzed by $t$-test. Statistical analyses were performed using a commercially available package - GraphPad Prism 6 (GraphPad Software Inc., San Diego, CA, USA). Significant differences were denoted as $\mathrm{p}<0.05$. Data were expressed as mean \pm standard error of the mean. All data in each group were first subjected to a Thompson's rejection test to eliminate outliers $(\mathrm{p}<0.01)$ [17], and the remaining data were used for the analysis among groups. The number of chicks per group ( $n=6-10)$, which was used in the current study generally considered as sufficient for statistical analysis, like one- or two-way ANOVA or $t$-test.

\section{RESULTS}

Rectal temperature, body weight and thyroid hormone concentrations at hatching

In ovo administration of L-Leu dose-dependently decreased $(\mathrm{p}<0.05) \mathrm{RT}$ at hatching in comparison with control-administered chicks. RT was found to have significantly decreased in the high-dose L-Leu group, and it showed a tendency to decrease $(p=0.078)$ in the low-dose L-Leu group, compared with the control group (Figure 1A). However, in ovo administration of L-Leu did not affect the relative BW of chicks at hatching (Figure $1 \mathrm{~B}$ ). The thyroid hormone $\mathrm{T}_{3}$ was not affected by in ovo administration of L-Leu (Figure 2A). However, in ovo administration of a high dose of L-Leu significantly $(\mathrm{p}<0.05)$ reduced the plasma $\mathrm{T}_{4}$ concentration and increased $(\mathrm{p}<0.05)$ the ratio of $\mathrm{T}_{3} / \mathrm{T}_{4}$ compared with the control group and the L-Leu (low-dose) group (Figures 2B, 2C).
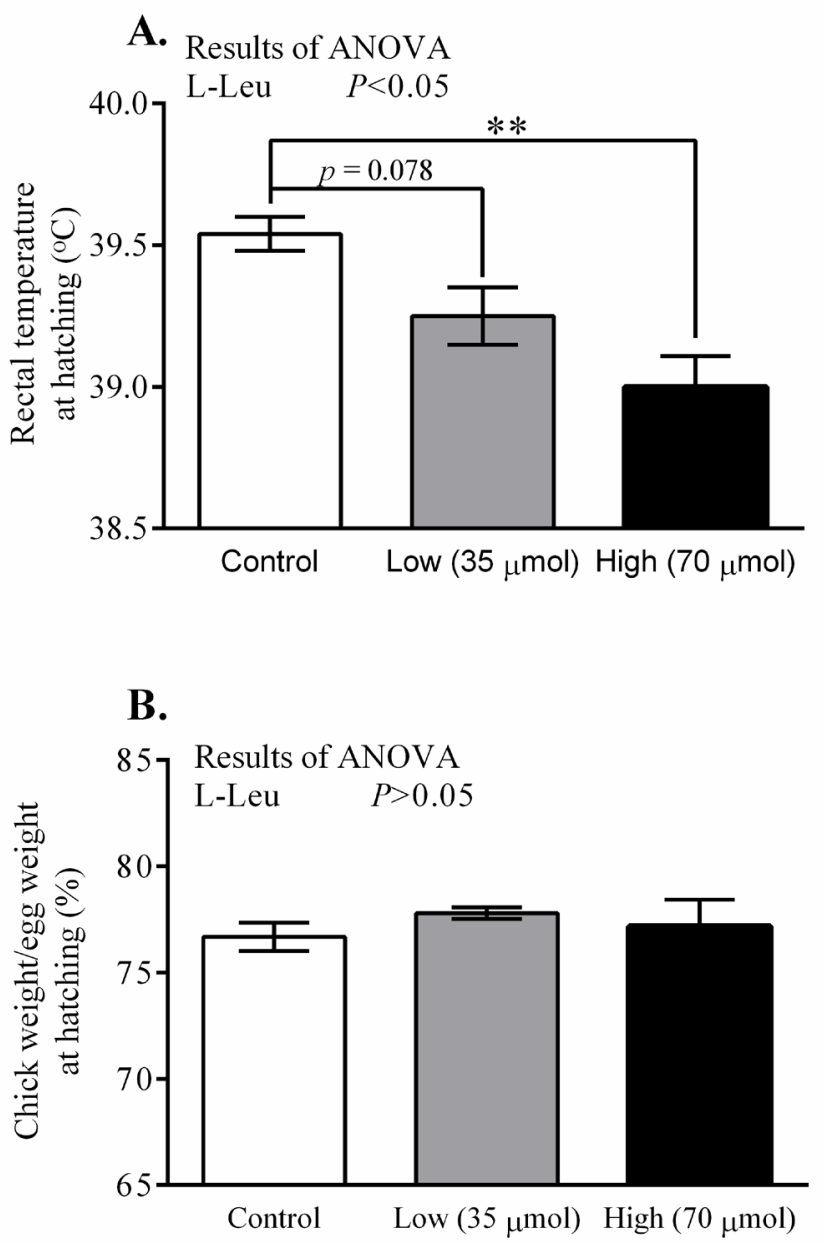

Figure 1. Effects of in ovo administration of L-Leu on rectal temperature (A) and the ratio of chick weight to egg weight (B) at hatching. The number of chicks in each group was as follows: control, $n=6$; low, $n=10 ;$ high, $n=9$. Values are expressed as mean \pm standard error of the mean. L-Leu, L-leucine; control, sterile water injection; low $(35 \mu \mathrm{mol})$, L-Leu injection with $35 \mu \mathrm{mol} / \mathrm{egg}$; high $(70 \mu \mathrm{mol})$, L-Leu injection with $70 \mu \mathrm{mol} / \mathrm{egg} .{ }^{* *} \mathrm{p}<0.01$ by Holm-Sidak's multiple comparisons test. 
A.

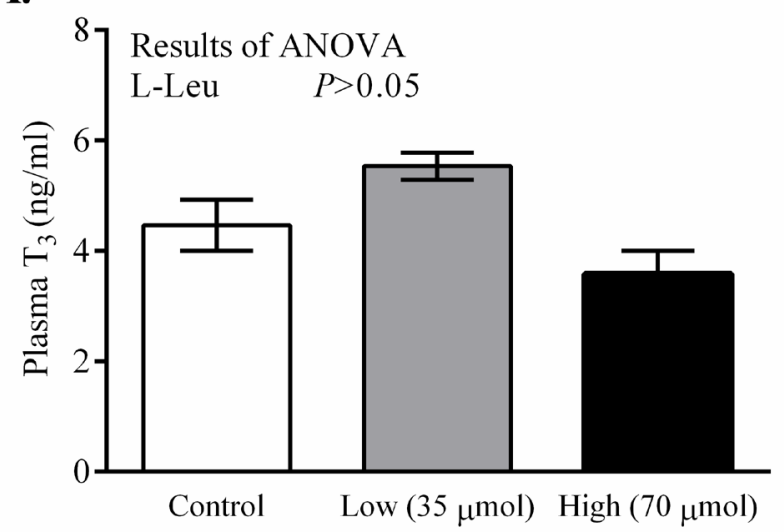

B.

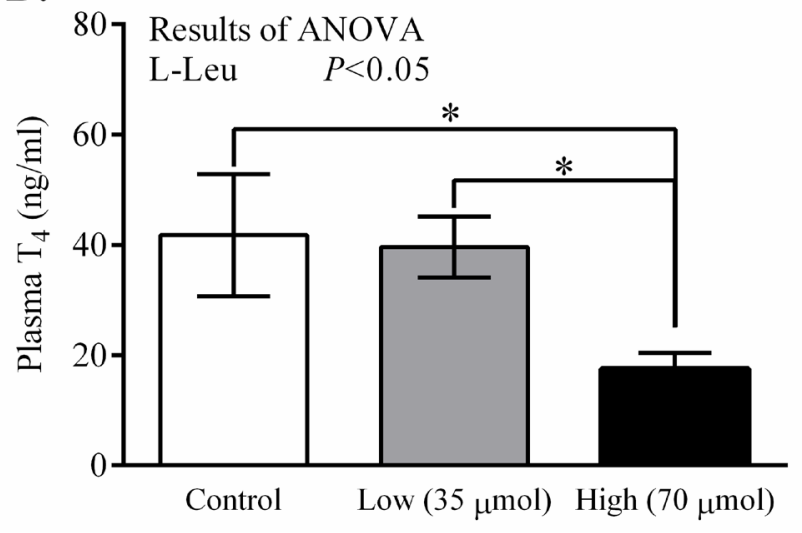

C.

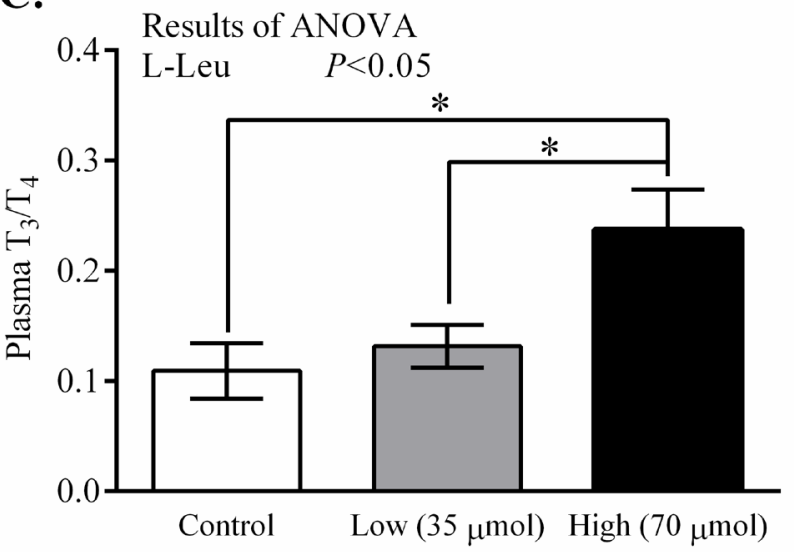

Figure 2. Effects of in ovo administration of L-Leu on plasma $T_{3}(A), T_{4}(B)$, and $\mathrm{T}_{3} / \mathrm{T}_{4}$ ratio $(C)$ at hatching. The number of chicks in each group was as follows: control, $n=6$; low, $n=10$; high, $n=9$. Values are expressed as mean \pm standard error of the mean. L-Leu, L-leucine; control, sterile water injection; low (35 $\mu \mathrm{mol})$, L-Leu injection with $35 \mu \mathrm{mol} / \mathrm{egg}$; high $(70 \mu \mathrm{mol})$, L-Leu injection with $70 \mu \mathrm{mol} /$ egg. * $p<0.05$ by Holm-Sidak's multiple comparisons test.

Changes in rectal temperature and body weight under $\mathrm{CT}$, as well as in RT, food intake and plasma metabolites under $\mathrm{HT}$, in post-hatched broiler chicks The RT and BW significantly $(\mathrm{p}<0.0001)$ increased with age from 3 days old, but the values for RT were constant from 4 days old. In ovo administration of L-Leu significantly increased
RT, but not BW gain, compared with the control treatment within the experimental days (Figure 3). HT caused RT to increase significantly $(\mathrm{p}<0.001)$ in broiler chicks. Notably, RT was significantly $(\mathrm{p}<0.05)$ lower in chicks administered with L-Leu in ovo than it was in control chicks administered in ovo under heat stress (Figure 4A), while food intake was not affected by in ovo administration of L-Leu under HT (Figure 4B). Plasma NEFA, $\mathrm{NH}_{3}$, LA, LDH, IgA, and ketone bodies were also not affected by in ovo administration of L-Leu in the current study (Table 1).

\section{DISCUSSION}

In the current study, we administered two doses (35 and 70 $\mu \mathrm{mol} / \mathrm{egg}$ ) of L-Leu in ovo and examined their effects on RT,

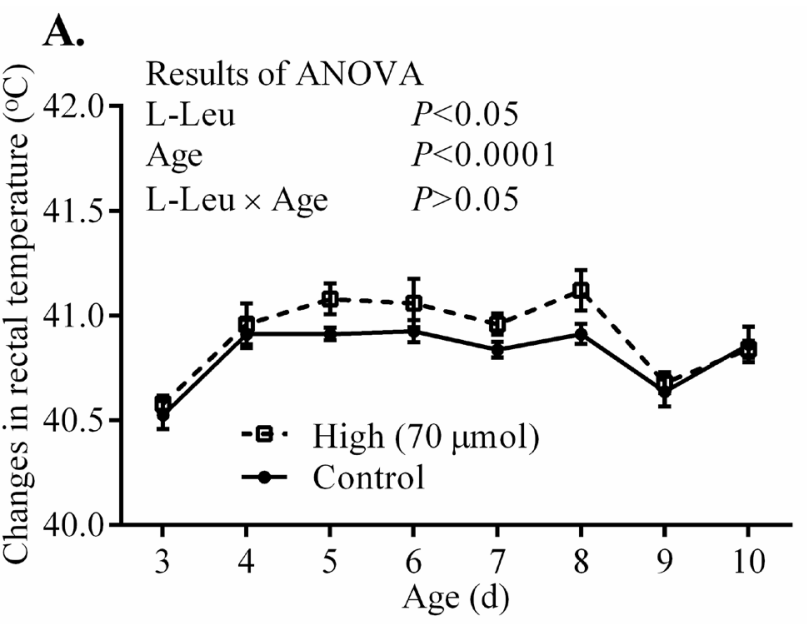

B.

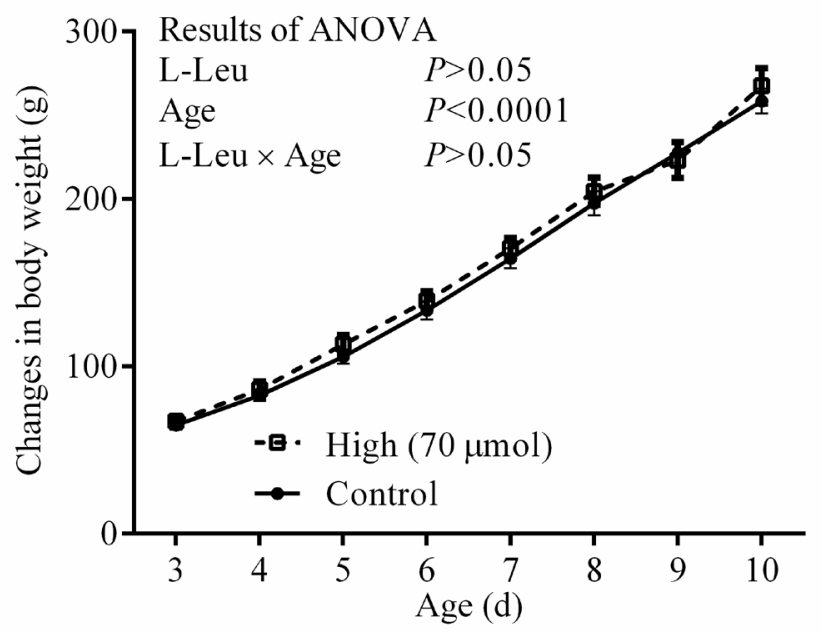

Figure 3. Effects of in ovo administration of L-Leu on changes in daily rectal temperature (A) and body weight (B) in post-hatched male broiler chicks. The number of chicks in each group was as follows: rectal temperature (control $=8$; high =6); body weight (control = 10; high =8). Values are expressed as mean \pm standard error of the mean. L-Leu, L-leucine; control, sterile water injection; high $(70 \mu \mathrm{mol})$, L-Leu injection with $70 \mu \mathrm{mol} / \mathrm{egg}$. 

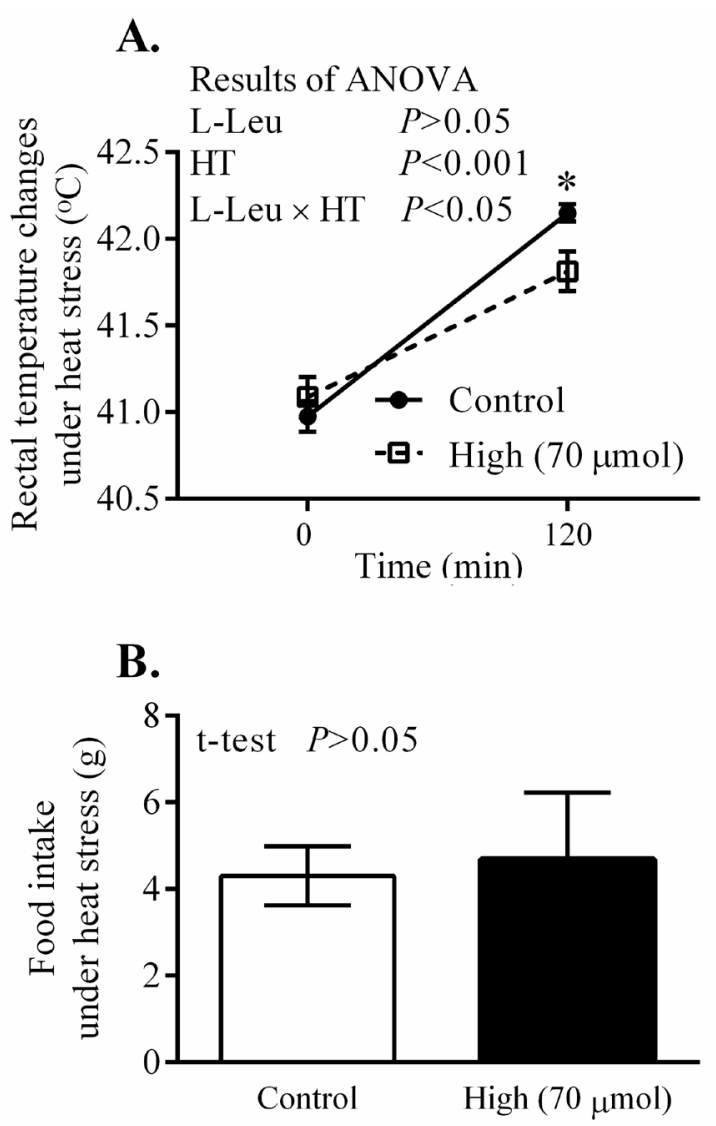

Figure 4. Effects of in ovo administration of L-Leu on changes in rectal temperature (A) and food intake (B) in heat-exposed male broiler chicks. The number of chicks in each group was $n=8$. Values are expressed as mean \pm standard error of the mean. L-Leu, L-leucine; HT, high ambient temperature $\left(35^{\circ} \mathrm{C} \pm 1^{\circ} \mathrm{C}\right)$; control, sterile water injection; high (70 $\mu \mathrm{mol})$, L-Leu injection with $70 \mu \mathrm{mol} / \mathrm{egg}$.

BW, and thyroid hormone levels at hatching in Experiment 1. The effects of high-dose administration of L-Leu (70 $\mu \mathrm{mol} / \mathrm{egg})$ on changes in daily RT and BW gain under CT, as well as on thermotolerance and food intake under HT, were examined in post-hatched chicks in Experiment 2. Accordingly, we confirmed that in ovo administration of a high dose of L-Leu has a stronger influence on regulation of body temperature at hatching. To the best of our knowledge, this is the first report to show that daily body temperature is higher in post-hatched chicks as a result of the in ovo administration of an amino acid-LLeu. The plasma metabolites were also confirmed not to have contributed to the L-Leu-mediated improvement in thermo- tolerance.

Recently, we reported that in ovo administration of L-Leu ( $35 \mu \mathrm{mol} / \mathrm{egg}$ ) once, on $\mathrm{ED} \mathrm{7,} \mathrm{has} \mathrm{a} \mathrm{strong} \mathrm{influence} \mathrm{in} \mathrm{terms}$ of stimulating embryonic metabolic activity and providing thermotolerance in post-hatched male broiler chicks under heat stress $[7,8]$. In the current study, males and females were not separated at hatching since sexing was conducted using the feather-identification method, which is only possible at 2 days old at the earliest. In ovo administration of L-Leu dosedependently decreased RT at hatching. The tendency that was observed ( $p=0.078)$ for RT to decline in the low-dose group compared with the control group might have been due to the sex-specific effects of in ovo administration of L-Leu, since it has been reported that females are more responsive to L-Leu administration in terms of showing a reduced RT at hatching [7]. At hatching, RT was clearly reduced in the high-dose group $(\mathrm{p}<0.01)$ compared with control chicks (Figure 1A). It has been reported that TM-dependent lower RT occurred at hatching due to a lower metabolic rate before hatching [18], which resulted from a feedback of higher metabolic rate under TM during embryogenesis [9]. Previously, we found that in ovo administration of L-Leu on ED 7 stimulated metabolic activity and lipid metabolism during the middle stages of embryogenesis [8]. Therefore, it could be hypothesized that the metabolic rate might be reduced before hatching due to the feedback of a higher metabolic rate in the middle of embryogenesis as a result of in ovo administration of L-Leu, which resulted in a lower RT at hatching in birds administered with L-Leu in ovo.

Thyroid hormones $\left(\mathrm{T}_{4}\right.$ and $\left.\mathrm{T}_{3}\right)$ increase with the process of embryonic development and reach a peak before hatching $[13,15]$. Both $T_{4}$ and $T_{3}$ have been documented to stimulate the metabolic rate in birds, except at the neonatal stage, and to play a role in heat production as well as in adaptive variation in heat exposure $[19,20]$. In the current study, the plasma $\mathrm{T}_{4}$ concentration decreased in association with lower body temperature in the high-dose group in comparison with control birds at hatching, and this finding was similar to that in a previous study, where the TM-mediated thyroid hormone and body temperature were found to have declined at hatching $[15,21]$. It has been reported that the thyroid and the adrenal axis contribute to thermal regulation in neonatal chicks. Although intraperitoneal injection of $\mathrm{T}_{3}$ and $\mathrm{T}_{4}$ did not influence body temperature, the inhibition of the hypothalamus-pi-

Table 1. Effects of in ovo administration of L-leucine on plasma metabolites in heat-exposed 10-days old male broiler chicks in Experiment 2

\begin{tabular}{lcccccc}
\hline Items & LA $(\mathbf{m g} / \mathbf{d L})$ & LDH $(\mathbf{m g} / \mathbf{d L})$ & NEFA $(\mathbf{m E q} / \mathbf{L})$ & IgA $(\mathbf{m g} / \mathbf{d L})$ & NH $_{3}(\mathbf{m E q} / \mathbf{L})$ & Ketone body $(\mathrm{mmol} / \mathbf{L})$ \\
\hline Control & $45.5 \pm 3.3$ & $560 \pm 39$ & $319 \pm 42$ & $18.5 \pm 0.11$ & $225 \pm 7$ & $404 \pm 38$ \\
High $(70 \mu \mathrm{mol})$ & $52.8 \pm 5.6$ & $527 \pm 26$ & $406 \pm 24$ & $18.5 \pm 0.09$ & $245 \pm 11$ & $428 \pm 29$ \\
p-value & 0.273 & 0.512 & 0.116 & 0.754 & 0.154 & 0.635 \\
\hline
\end{tabular}

The number of chicks used in each group was 7 to 9 . Values are means \pm standard error of the mean.

LA, lactic acid; LDH, lactate dehydrogenase; NEFA, non-esterified fatty acid; IgA, immunoglobulin A; $\mathrm{NH}_{3}$, ammonia; High (70 $\mu$ mol), L-Leu injection with $70 \mu$ mol/egg. 
tuitary-adrenal axis was found to lower body temperature [22]. Despite being homoeothermic creatures, neonatal chicks cannot keep their body temperature constant unless the environmental temperature is warmed [14]. This may be explained by the insufficient function of the thyroid hormones during the neonatal stage. TM during embryogenesis caused the function not only of the thyroid but also of the adrenal axis to decline, and resulted in hypothermia [12]. This hypothermia was mainly due to the reduced function of the adrenal axis [22]. Moreover, the function of $\mathrm{T}_{4}$ is weaker than that of $\mathrm{T}_{3}$ [14]. In addition, our recent studies showed that the concentration of plasma thyroid hormones $\left(\mathrm{T}_{4}\right.$ and $\left.\mathrm{T}_{3}\right)$ was not affected by in ovo administration of L-Leu under short-term heat stress, and that even thermotolerance was improved in 4-week-old male broilers (unpublished data). Together, these findings suggest that the reduced body temperature found in the high-dose group at hatching might not be explained by the lower level of plasma $\mathrm{T}_{4}$.

After hatching, body temperature and BW gain increased with the progress of age in neonatal chicks $[7,8,23]$. Chicks, which are precocial birds, have a limited initial ability to maintain homeostasis of body temperature at hatching [14]; however, the thermoregulatory functions become well developed and are mature at 10 days old in broilers [24]. The higher level of body temperature in neonatal broiler chicks administered with L-Leu in ovo suggests that their thermoregulatory functions might have developed well or become mature earlier compared with control broilers. The higher body temperature might have been aided by the relatively high metabolic rate in chicks administered with L-Leu in ovo. However, the unaffected BW gain in chicks administered with L-Leu in ovo suggests that their food intake could be expected to have increased simply to support the high metabolic rate. Food intake and plasma thyroid hormones in post-hatched chicks need to be investigated in future studies.

Heat exposure significantly increased body temperature, which is a normal physiological response under heat stress [16]. Increased body temperature is beneficial for increasing sensible heat loss; however, it has been assumed that sensible heat loss does not play an important role when the ambient temperature is above the upper limit of the thermoneutral zone [25]. Prolonged heat exposure and high body temperature cause oxidative stress $[3,26]$ in chickens. Body temperature is a reliable parameter of thermotolerance acquisition in avians [14]. Body temperature was significantly lowered in chicks administered with L-Leu in ovo compared with control chicks under heat exposure (Figure 4A), which suggests that in ovo administration of L-Leu improved the thermotolerance of chicks $[7,8,27]$. Higher heat loss or lower heat production, or both, contribute to lowering body temperature [28]. A previous study showed that food intake was reduced and lipid metabolism was activated after $180 \mathrm{~min}$ of heat exposure, and also that body temperature was significantly lower at $120 \mathrm{~min}$ of a heat challenge, in chicks administered with L-Leu in ovo $[7,8]$. In the current study, body temperature was significantly reduced at $120 \mathrm{~min}$ of heat stress in chicks administered with L-Leu in ovo in comparison with control birds; however, food intake and plasma metabolites (NEFA, ketone bodies) were not affected at $120 \mathrm{~min}$ of heat exposure (Figure 4B, Table 1). These results indicate that food intake and lipid metabolism might not be the main contributors to L-Leu-mediated improvement in thermotolerance. Our recent study also supports the finding that thermotolerance was improved without affecting food intake and lipid metabolism after $120 \mathrm{~min}$ of heat stress in 4-week-old broilers administered with L-Leu in ovo (unpublished data). Therefore, it could be speculated that in ovo administration of L-Leu might cause some epigenetic modulations in certain neural factors in broiler chicks to afford thermotolerance since the brain is the center of thermoregulation [14]. Future research will aim to clarify this issue.

In conclusion, the stronger effects of high-dose in ovo administration of L-Leu caused hypothermia and a reduced plasma $\mathrm{T}_{4}$ level at hatching, as well as enhancing the daily thermoregulation process in neonatal chicks and affording thermotolerance. The present study clarified that food-intake regulation and lipid metabolism did not contribute to the L-Leu-mediated improvement in thermotolerance under shortterm heat stress. A future study will reveal the biochemical and molecular mechanisms whereby L-Leu improves thermotolerance.

\section{CONFLICT OF INTEREST}

We certify that there is no conflict of interest with any financial organization regarding the material discussed in the manuscript.

\section{ACKNOWLEDGMENTS}

This study was supported by JSPS KAKENHI Grant Numbers JP15K07694 and JP18K19271 to VSC and JP17KT0077 to TB.

\section{REFERENCES}

1. Geraert PA, Padilha JCF, Guillaumin S. Metabolic and endocrine changes induced by chronic heatexposure in broiler chickens: growth performance, body composition and energy retention. Br J Nutr 1996;75:195-204.

2. Azad MA, Kikusato M, Zulkifli I, Toyomizu M. Electrolysed reduced water decreases reactive oxygen species-induced oxidative damage to skeletal muscle and improves performance in broiler chickens exposed to medium-term chronic heat stress. Br Poult Sci 2013;54:503-9.

3. Chowdhury VS, Tomonaga S, Ikegami T, et al. Oxidative damage 
and brain concentrations of free amino acid in chicks exposed to high ambient temperature. Comp Biochem Physiol A Mol Integr Physiol 2014;169:70-6.

4. Ito K, Erwan E, Nagasawa M, Furuse M, Chowdhury VS. Changes in free amino acid concentrations in the blood, brain and muscle of heat-exposed chicks. Br Poult Sci 2014;55:644-52.

5. Chowdhury VS, Shigemura A, Erwan E, et al. Oral administration of L-citrulline, but not L-arginine or L-ornithine, acts as a hypothermic agent in chicks. J Poult Sci 2015;52:331-5.

6. Chowdhury VS, Han G, Bahry MA, et al. L-Citrulline acts as potential hypothermic agent to afford thermotolerance in chicks. J Therm Biol 2017;69:163-70.

7. Han G, Yang H, Bahry MA, et al. L-Leucine acts as a potential agent in reducing body temperature at hatching and affords thermotolerance in broiler chicks. Comp Biochem Physiol A Mol Integr Physiol 2017;204:48-56.

8. Han $\mathrm{G}$, Yang $\mathrm{H}$, Bungo T, et al. In ovo L-leucine administration stimulates lipid metabolisms in heat-exposed male, but not female, chicks to afford thermotolerance. J Therm Biol 2018; 71:74-82.

9. Chowdhury VS. Heat stress biomarker amino acids and neuropeptide afford thermotolerance in chicks. J Poult Sci 2019;56: 1-11.

10. Kim DH, Kim SH, Jeong WS, Lee HY. Effect of BCAA intake during endurance exercises on fatigue substances, muscle damage substances, and energy metabolism substances. J Exerc Nutr Biochem 2013;17:169-80.

11. Green CR, Wallace M, Divakaruni AS, et al. Branched-chain amino acid catabolism fuels adipocyte differentiation and lipogenesis. Nat Chem Biol 2016;12:15-21.

12. Piestun Y, Shinder D, Ruzal M, Halevy O, Yahav S. The effect of thermal manipulations during the development of the thyroid and adrenal axes on in-hatch and post-hatch thermoregulation. J Therm Biol 2008;33:413-8.

13. McNabb FMA, Darras VM. Thyroids. In: Scanes CG, editor. Sturkie's avian physiology. 6th ed. Milwaukee, WI, USA: Academic Press; 2015. p. 535-47.

14. Yahav S. Regulation of body temperature: strategies and mechanisms. In: Scanes CG, editor. Sturkie's avian physiology. 6th ed. Milwaukee, WI, USA: Academic Press; 2015. p. 869-905.

15. Piestun Y, Halevy O, Yahav S. Thermal manipulations of broiler embryos-the effect on thermoregulation and development during embryogenesis. Poult Sci 2009;88:2677-88.
16. Chowdhury VS, Tomonaga S, Nishimura S, Tabata S, Furuse M. Physiological and behavioral responses of young chicks to high ambient temperature. J Poult Sci 2012;49:212-8.

17. Kobayashi K, Pillai KS. Transformation of data and outliers. In: Kobayashi K, Pillai, KS, editors. A handbook of applied statistics in pharmacology. Boca Raton, FL, USA: CRC Press; 2013. p. 37-46.

18. Piestun Y, Zimmerman I, Yahav S. Thermal manipulations of turkey embryos: the effect on thermoregulation and development during embryogenesis. Poult Sci 2015;94:273-80.

19. Danforth E, Burger A. The role of thyroid hormones in the control of energy expenditure. Clin Endocrinol Metab 1984;13: 581-95.

20. Wentworth BC, Ringer RK. Thyroids. In: Sturkie PD, editor. Avian physiology. New York, USA: Springer; 1986. p. 452-65.

21. Willemsen H, Kamers B, Dahlke F, et al. High- and low-temperature manipulation during late incubation: effects on embryonic development, the hatching process, and metabolism in broilers. Poult Sci 2010;89:2678-90.

22. Takahashi H, ligo M, Ando K, et al. Regulation of body temperature by thyrotropin-releasing hormone in neonatal chicks. Dev Brain Res 2005;157:58-64.

23. De Basilio V, Requena F, Leon A, Vilarino M, Picard M. Early age thermal conditioning immediately reduces body temperature of broiler chicks in a tropical environment. Poult Sci 2003; 82:1235-41.

24. Arad Z, Itsaki-Glucklish S. Ontogeny of brain temperature in quail chicks (Coturnix coturnix japonica). Physiol Zool 1991; 64:1356-70.

25. Hillman PE, Scott NR, Van Tienhoven A. Physiological responses and adaptations to hot and cold environments. In: Yousef MK, editor. Stress physiology in livestock. Boca Raton, FL, USA: CRC Press; 1985. p. 27-71.

26. Mujahid A, Pumford NR, Bottje W, et al. Mitochondrial oxidative damage in chicken skeletal muscle induced by acute heat stress. J Poult Sci 2007;44:439-45.

27. Tanizawa H, Shiraishi JI, Kawakami SI, Tsudzuki M, Bungo T. Effect of short-term thermal conditioning on physiological and behavioral responses to subsequent acute heat exposure in chicks. J Poult Sci 2014;51:80-6.

28. Aggarwal A, Upadhyay R. Heat stress and animal productivity. 1st ed. India: New Delhi, India: Springer; 2012. 